\title{
Extensive genomic diversity of closely related Wolbachia strains
}

Correspondence

Julie C. Dunning Hotopp

jdhotopp@som.umaryland.edu

Received 16 January 2009

Revised 24 March 2009

Accepted 25 March 2009
Nadeeza Ishmael, ${ }^{1,2}$ † Julie C. Dunning Hotopp, ${ }^{1,2} \dagger$ Panagiotis loannidis, ${ }^{3}$ Sarah Biber, ${ }^{4} \S$ Joyce Sakamoto, ${ }^{2}$ Stefanos Siozios, ${ }^{3}$ Vishvanath Nene, ${ }^{1,2} \|$ John Werren, ${ }^{5}$ Kostas Bourtzis, ${ }^{3}$ Seth R. Bordenstein ${ }^{4,6}$ and Hervé Tettelin ${ }^{1,2}$

\author{
1J. Craig Venter Institute, 9708 Medical Center Dr., Rockville, MD 20850, USA \\ ${ }^{2}$ Institute for Genome Sciences, Department of Microbiology and Immunology, University of \\ Maryland Baltimore, Baltimore, MD 21201, USA \\ ${ }^{3}$ Department of Environmental and Natural Resources Management, University of loannina, 30100 \\ Agrinio, Greece \\ ${ }^{4}$ Josephine Bay Paul Center for Comparative Molecular Biology and Evolution, The Marine \\ Biological Laboratory, Woods Hole, MA 02543, USA \\ ${ }^{5}$ Biology Department, University of Rochester, Rochester, NY 14627, USA \\ ${ }^{6}$ Department of Biological Sciences, Vanderbilt University, Nashville, TN 37235, USA
}

Using microarray-based comparative genome hybridization (mCGH), the genomic content of Wolbachia pipientis wMel from Drosophila melanogaster was compared to the closely related Wolbachia from $D$. innubila (wlnn), D. santomea ( $w S a n)$, and three strains from $D$. simulans ( $w A u$, $w \mathrm{Ri}, w \mathrm{Sim})$. A large number of auxiliary genes are identified in these five strains, with most absent/ divergent genes being unique to a given strain. Each strain caused an average of $\sim 60$ genes to be removed from the core genome. As such, these organisms do not appear to have the streamlined genomes expected of obligate intracellular bacteria. Prophage, hypothetical and ankyrin repeat genes are over-represented in the absent/divergent genes, with 21-87\% of absent/divergent genes coming from prophage regions. The only wMel region absent/divergent in all five query strains is that containing WD_0509 to WD_0511, including a DNA mismatch repair protein MutL-2, a degenerate RNase, and a conserved hypothetical protein. A region flanked by the two portions of the WO-B prophage in wMel is found in four of the five Wolbachia strains as well as on a plasmid of a rickettsial endosymbiont of Ixodes scapularis, suggesting lateral gene transfer between these two obligate intracellular species. Overall, these insect-associated Wolbachia have highly mosaic genomes, with lateral gene transfer playing an important role in their diversity and evolution.

\section{INTRODUCTION}

Wolbachia bacteria are common obligate intracellular endosymbionts that infect a wide variety of invertebrates,

TThese authors contributed equally to this paper.

łPresent address: Centre for Structural and Functional Genomics, Concordia University, Montréal, QC H4B 1R6, Canada.

§Present address: Howard Hughes Medical Institute and Department of Biology, Brandeis University, Waltham, MA 02454, USA.

||Present address: International Livestock Research Institute, PO Box 30709, Nairobi 00100, Kenya.

Abbreviation: $\mathrm{mCGH}$, microarray comparative genome hybridization. The GEO series accession number for the microarray data of this study is GSE13825. including arthropods and filarial nematodes. The arthropod-infecting Wolbachia strains are maternally inherited and exert unusual effects on host reproduction, including: (1) parthenogenesis, whereby infected virgin females produce infected female offspring, (2) male killing, whereby infected male embryos fail to develop, (3) feminization, whereby genetic males develop into reproductively capable females, and (4) cytoplasmic incompatibility, the most common phenotype, whereby the offspring of uninfected females and infected males fail to develop (Stouthamer et al., 1999; Werren et al., 2008). These phenotypes increase the number of infected hosts within a population, promoting the frequency of Wolbachia. Although insect-associated Wolbachia are typically considered reproductive parasites, some 
Wolbachia strains have no reproductive phenotype (Hoffmann et al., 1996; Turelli \& Hoffmann, 1995), and mutualistic host-bacteria interactions have also been described (e.g. Dobson et al., 2002; Hedges et al., 2008; Teixeria et al., 2008). Phylogenetic analysis of concatenated protein sequences and the 16S rRNA places Wolbachia in a clade of obligate intracellular bacteria including the tickand trematode-vectored vertebrate pathogens from the genera Anaplasma, Ehrlichia and Neorickettsia, as well as the more distantly related, but better characterized, arthropod-associated Rickettsia spp. (Dumler et al., 2001; Dunning Hotopp et al., 2006b). Numerous phylogenetic analyses of Wolbachia have been undertaken, including single-locus and multi-locus approaches (Bordenstein et al., 2008; Casiraghi et al., 2005; Lo et al., 2002; O’Neill et al., 1992; Werren et al., 1995; Zhou et al., 1998). Despite maternal inheritance in arthropods, the phylogenetic distribution of these Wolbachia is not concordant with that of their hosts (Baldo et al., 2006b; O'Neill et al., 1992; Werren et al., 1995). Therefore, in arthropods both horizontal transmission of Wolbachia and loss of infection are likely, although the mechanisms are not fully understood (Bouchon et al., 1998; Heath et al., 1999; Huigens et al., 2004)

Species designations in Wolbachia have remained elusive, largely owing to the lack of an accepted definition of bacterial species that can be applied to obligate intracellular bacteria. However, current phylogenetic and recombination analysis supports all Wolbachia strains being formally included in the single species Wolbachia pipientis (Lo et al., 2007). To help distinguish isolates, Wolbachia strains have been divided into eight phylogenetic clades termed supergroups (Bordenstein et al., 2008; Lo et al., 2007). The A and B supergroup Wolbachia are the most common and are widespread in arthropods as single or double infections (Werren et al., 1995). The C and D supergroups contain the Wolbachia strains from filarial nematodes (Lo et al., 2002). Primitive wingless insects (springtails; Collembola) are infected with supergroup E (Czarnetzki \& Tebbe, 2004; Lo et al., 2002; Vandekerckhove et al., 1999). Supergroup F consists of Wolbachia infecting termites, weevils, true bugs and filarial nematodes (Casiraghi et al., 2001; Lo et al., 2002; Rasgon \& Scott, 2004). Supergroup H infects termites (Bordenstein \& Rosengaus, 2005). Three other divergent lineages exist that have not been given supergroup designations, including the infection found in the flea Ctenocephalides canis (Casiraghi et al., 2004), the filarial nematode Dipetalonema gracile (Casiraghi et al., 2004), and the pseudoscorpion Cordylochernes scorpioides (Zeh et al., 2005; Zeh \& Zeh, 2006). Double or triple infections have been described with isolates coming from different or identical supergroups (Werren et al., 1995).

Obligate intracellular bacteria are often viewed as excellent model systems to understand the stable, symbiotic interactions that occur between prokaryotes and their eukaryotic hosts. However, recent analyses of sequenced genomes of host-switching obligate intracellular bacteria, like Wolbachia, suggest that mobile elements and genetic exchange are not as rare as previously thought (Bordenstein \& Reznikoff, 2005). The presence of extensive recombination (Baldo et al., 2006a; Klasson et al., 2009), mobile elements (Klasson et al., 2008; Wu et al., 2004) and lateral phage transfer between co-infecting strains (Bordenstein \& Wernegreen, 2004; Masui et al., 2000) facilitates high levels of genetic exchange in Wolbachia. These processes that shape the Wolbachia genome are likely influenced by exposure to novel gene pools available due to horizontal transmission of infection, multiple infections and large numbers of infected hosts.

The high level of genetic exchange in Wolbachia suggests that like many free-living bacteria, Wolbachia has a core genome complemented by an extensive auxiliary genome. A core genome consists of all the genes shared by all (or almost all) sequenced strains for a given taxon, typically a species (Boucher et al., 2001; Hogg et al., 2007; Lan \& Reeves, 2000; Tettelin et al., 2005, 2008). All of the other genes constitute what has been termed the dispensable (Tettelin et al., 2005, 2008), contingency (Hogg et al., 2007) or auxiliary (Boucher et al., 2001; Lan \& Reeves, 2000) genome, which encompasses the genetic variation within the species. A subset of the auxiliary genes includes the unique (Hogg et al., 2007) or strain-specific genes (Tettelin et al., 2005, 2008), those genes found only in a single genome of the species. When the auxiliary genome is combined with the core genome, the result is the pangenome (Tettelin et al., 2005, 2008) or supragenome (Hogg et al., 2007). Pan-genomes can be classed as open/ unbounded or closed/bounded (Medini et al., 2005; Tettelin et al., 2008). With an open pan-genome, each additional genome sequenced, on average, contributes new genes to the pan-genome. In contrast, with a closed pangenome, each additional genome sequenced contributes no new genes to the pan-genome. Species with open pangenomes include Streptococcus agalactiae, Streptococcus pneumoniae, Escherichia coli, Bacillus cereus and Prochlorococcus marinus (Tettelin et al., 2008). Those with closed pan-genomes include Staphylococcus aureus, Streptococcus pyogenes, Ureaplasma urealyticum and Bacillus anthracis (Tettelin et al., 2008). In addition to the above nomenclature, another convention has been proposed that combines all genes found in $>1 \%$ of strains into a species genome, and any genes in $<1 \%$ of strains are considered of foreign origin or on the decline (Boucher et al., 2001; Lan \& Reeves, 2000).

In order to examine the core genome and the genetic flux between $W$. pipientis strains, we examined the genomic content of five strains using microarray-based comparative genomic hybridization (mCGH) on a Wolbachia microarray. DNAs from reference and query organisms are differentially labelled and competitively hybridized to a microarray (Behr et al., 1999). The hybridization data reveal the presence/absence of genes in the query genome relative to the genome content of the reference organism. For Wolbachia, a large number of auxiliary genes are 
identified in five closely related supergroup A strains, with most absent/divergent genes unique to a given strain. Prophage, hypothetical and ankyrin repeat genes are overrepresented in the absent/divergent genes, with 3-55 of those being unique to a given strain. One variable region flanked by the two portions of the WO-B prophage in $w \mathrm{Mel}$ is also identified in a plasmid of a Rickettsia endosymbiont of Ixodes scapularis (Noda et al., 1997) suggesting that lateral gene transfer extends across the order Rickettsiales.

\section{METHODS}

Bacterial strains. Genomic DNA from six Wolbachia strains was hybridized in this study (Table 1). These are a subset of well-studied supergroup A strains that were chosen because they were expected to be genetically similar but induce a variety of reproductive phenotypes. The supergroup A Wolbachia strains from Nasonia vitripennis 12.1, Drosophila recens, D. yakuba and D. teissieri were also initially chosen for inclusion, but were later removed because of difficulties in background hybridization, likely due to host DNA contamination.

Microarray construction. A $w \mathrm{Mel}$ microarray was constructed which represents $95 \%$ of the unique ORFs (Wu et al., 2004). Primers were designed from the unique predicted genes annotated in $w$ Mel. In order to obtain pure amplicons devoid of background amplification of host genes, all amplicons were produced, where possible, from two separate clones from the $w \mathrm{Mel}$ genome-sequencing project. Genes in physical gaps where a sequencing clone was not available were amplified directly from genomic DNA from Drosophila melanogaster infected with $w \mathrm{Mel}$. Amplicons (50-1000 bp) were produced using AmpliTaq (Applied Biosystems), purified using filtration plates (Millipore), and analysed on agarose gels. The purified amplicons were diluted $1: 1$ with DMSO, spotted in quadruplicate onto Corning UltraGap slides with a Lucidea printing robot (GE Healthcare) and irradiated with UV light (Dunning Hotopp et al., 2006a).

Genomic DNA preparation and whole-genome amplification. Wolbachia cells were isolated from 2-4 g of live, whole insects via density centrifugation, and whole chromosomal fragments were purified following pulsed-field gel separation as described by Wernegreen et al. (2002). Due to the small amounts of chromosomal fragments recovered, whole-genome amplification was needed to produce enough DNA for hybridization. Therefore $\sim 10 \mathrm{ng}$ of genomic DNA was amplified per reaction with GenomiPhi. A similar strategy was used successfully for mCGH studies in Anaplasma phagocytophilum (Dunning Hotopp et al., 2006b). Whole-genome amplification has been shown to be specific, with a randomly distributed bias and a mutation rate of $<2 \times 10^{-5}$ (Zhang et al.,
2006). In the obligate intracellular bacterium Ehrlichia chaffeensis Arkansas, a comparison of genomic DNA with GenomiPhi-amplified DNA also revealed a randomly distributed bias (data not shown). A similar comparison of genomic DNA to GenomiPhi-amplified DNA was not possible for Wolbachia DNA in the current study due to an inability to obtain large quantities of genomic DNA. However, a comparison of two independent GenomiPhi amplifications of the same genomic DNA sample from strain $w$ Ri demonstrates the reproducibility of the amplification (Fig. 1). To reduce the effects of any bias, each probe was synthesized from a pool of three distinct GenomiPhi amplifications.

Microarray hybridizations. Cy 3 and Cy5 probes were synthesized from GenomiPhi-amplified DNAs as described by Tettelin et al. (2001). Briefly, amino-allyl-dUTP-labelled probes were synthesized from $4 \mu \mathrm{g}$ of genomic DNA using Klenow fragment $\left(3^{\prime} \rightarrow 5^{\prime}\right.$ exo-) (New England Biolabs). The reactions were purified using the QIAquick PCR purification kit (Qiagen) with modified phosphate buffers. Cy3 or Cy5 dyes (GE Healthcare) were chemically coupled to the incorporated amino-allyl-dUTP in carbonate buffer. Probe pairs were resuspended in $5 \times$ SSC, $50 \%$ formamide, $0.1 \%$ SDS, $0.1 \mathrm{mM}$ DTT and $0.35 \mathrm{mg} \mathrm{ml}^{-1}$ salmon sperm DNA and hybridized to slides overnight at $42{ }^{\circ} \mathrm{C}$. The hybridized slides were washed twice in $2 \times$ SSC, $0.1 \%$ SDS at $55{ }^{\circ} \mathrm{C}$; twice in $0.1 \times$ SSC, $0.1 \%$ SDS at room temperature; twice in $0.1 \times$ SSC at room temperature; and once in MilliQ water at room temperature. They were then dried, and scanned using a GenePix4000B scanner (Molecular Devices).

Microarray data analysis and bioinformatics. Cy3- and Cy5labelled probes were synthesized from a pool of three distinct GenomiPhi amplifications to reduce bias. One flip-dye experiment (two hybridizations) was performed with each of three independent pools, yielding a total of six hybridizations per strain. With 4-8 printed replicates per slide this yielded 24-48 replicated spots per gene to compare across the study. Ratios were calculated as $w \mathrm{Mel}$ reference fluorescence over the query fluorescence (reference/query) and were normalized using iterative log-mode centring (Dunning Hotopp et al., 2006a). The geometric mean ratio was calculated for all good spots in each flip-dye experiment. The values reported are the medians of the geometric mean ratio for the three flip-dye experiments. Ideally, hybridization results would be compared to gene presence/absence from two closed genome sequences to establish microarray platformspecific criteria for absence/divergence. However, only one supergroup A Wolbachia has had its genome fully sequenced and closed. Therefore, previously established criteria for a Neisseria meningitidis microarray were used (Dunning Hotopp et al., 2006a), with genes deemed absent/ divergent (ratio $>3$ ), present (ratio $<2$ ), or not predictive (ratio $2-3$ ).

Southern hybridizations. Total genomic DNA from Drosophila lines was extracted according to the Holmes-Boner method (Sambrook et al., 1989). The DNA fragments generated from $5 \mu \mathrm{g}$

Table 1. Wolbachia strains used in this study

\begin{tabular}{|llll|}
\hline Strain & \multicolumn{1}{c}{ Host } & Reproductive phenotype & \multicolumn{1}{c|}{ Reference } \\
\hline$w \mathrm{Mel}$ & Drosophila melanogaster & Cytoplasmic incompatibility & Wu et al. (2004) \\
$w \mathrm{Inn}$ & Drosophila innubila & Male killer & Dyer \& Jaenike (2005) \\
$w \mathrm{Au}$ & Drosophila simulans & None & Hoffmann et al. $(1996) ;$ Turelli \& \\
& & & Hoffmann (1995) \\
$w \mathrm{Ri}$ & Drosophila simulans & Cytoplasmic incompatibility & Hoffmann et al. (1986) \\
$w \mathrm{Sim}$ & Drosophila simulans & Unknown & (Salzberg et al. (2005) \\
$w \mathrm{San}$ & Drosophila santomea & Cytoplasmic incompatibility & Zabalou et al. $(2004) ;$ Zabalou et al. \\
& & & (2008) \\
\hline
\end{tabular}




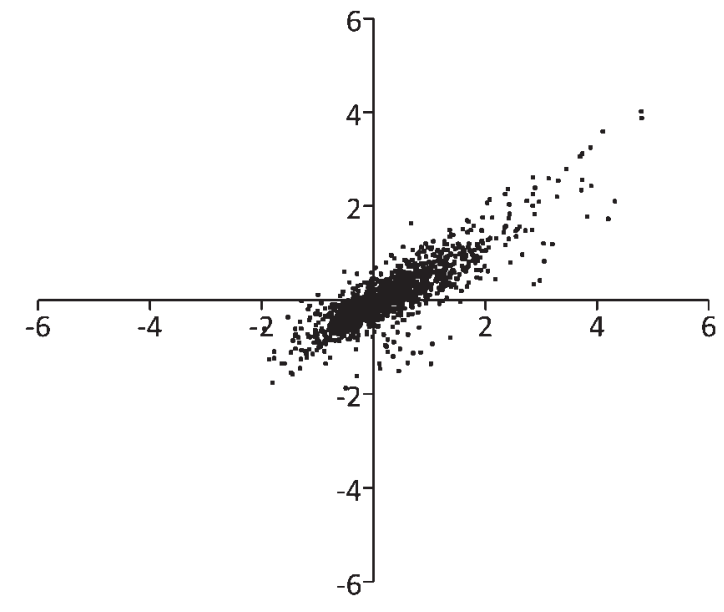

Fig. 1. Comparison of independent hybridizations of DNA from whole-genome amplifications. The ratios from two flip-dye experiments for strain $w R$ Ri are positively correlated, with $R^{2}=0.73145$, indicating the reproducibility of whole-genome amplification of the samples.

EcoRI-digested genomic DNA were separated on a $0.8 \%$ agarose gel and blotted onto Immobilon-Ny+ filter (Millipore) according to the manufacturer's instructions and hybridized at $68{ }^{\circ} \mathrm{C}$ for $18 \mathrm{~h}$. WD_0514 ANK probe was generated by PCR amplification with Aorf818F 5'-ATACAGTGGCCGGATAAATAAC-3' and Aorf818R 5'AGCACCAACAACTAAACTTCTCA- $3^{\prime}$ in $50 \mu$ reactions containing $50 \mathrm{mM} \mathrm{KCl}, 10 \mathrm{mM}$ Tris/HCl pH 9.0, 0.1\% Triton X-100, $1.5 \mathrm{mM}$ $\mathrm{MgCl}_{2}, 125 \mu \mathrm{M}$ dNTPs, $500 \mathrm{nM}$ of each primer, $1 \mathrm{U}$ Taq DNA polymerase (Promega) and $50 \mathrm{ng}$ DNA, with an initial denaturation step at $94{ }^{\circ} \mathrm{C}$ for $5 \mathrm{~min} ; 35$ cycles of $94{ }^{\circ} \mathrm{C}$ for $30 \mathrm{~s}, 56{ }^{\circ} \mathrm{C}$ for $30 \mathrm{~s}$, and $72{ }^{\circ} \mathrm{C}$ for $3 \mathrm{~min}$; and a final elongation step at $72{ }^{\circ} \mathrm{C}$ for $10 \mathrm{~min}$. PCR products were purified with a PCR purification kit (Qiagen) and radioactively labelled with $\left[\alpha^{32} \mathrm{P}\right] \mathrm{dATP}$ using the Prime-a-gene labelling kit (Promega). A specific probe for the Wolbachia fts $Z$ cell-division gene was also generated with the primers ftsZ1 5'-GTATGCCGATTGCAGAGCTTG- $3^{\prime}$ and ftsZ2 $5^{\prime}$-GCCATGAGTATTCACTTGGCT3' (Holden et al., 1993) and used as positive control for Wolbachia DNA.

PCR amplification. MLST profiles of $w$ San and $w$ Sim were obtained as previously described (Baldo et al., 2006b) using Taq (Promega) or HotStarTaq (Qiagen). The region from WD_0509 to WD_0514 was amplified using primers 467F (5' -TTCTTGATCTCACAATCTCTA-3') and 566R (5'-AGACTGTGCCTGAGAATCAA- $\left.3^{\prime}\right)$ with a $98{ }^{\circ} \mathrm{C}$ initial denaturation for $30 \mathrm{~s} ; 35$ cycles of $98{ }^{\circ} \mathrm{C}$ for $10 \mathrm{~s}, 55^{\circ} \mathrm{C}$ for $30 \mathrm{~s}, 72{ }^{\circ} \mathrm{C}$ for $20 \mathrm{~min}$; and a final $72{ }^{\circ} \mathrm{C}$ elongation for $10 \mathrm{~min}$ using either Elongase (Invitrogen) or Phusion (Finnzymes) according to the manufacturer's instructions with $1.5 \mathrm{mM} \mathrm{MgCl}_{2}$ and $2 \mathrm{mM}$ dNTPs. PCR products were purified with a PCR purification kit (Qiagen) or treated with shrimp alkaline phosphatase and exonuclease I (Amersham). Sequencing was performed by Macrogen and the Institute for Genome Sciences at the University of Maryland Baltimore.

\section{RESULTS}

\section{Core genome}

The core bacterial genome can be defined as those genes conserved among all bacterial strains of a given species. In this study, each query strain leads to the removal of $\sim 60$ genes on average from the core $W$. pipientis genome (Fig. 2B). An exponential regression suggests that the core genome consists of 621 genes. Although this estimate is based on only five data points, it is roughly consistent with the $743 w \mathrm{Mel}$ genes in the 637 shared clusters of orthologous gene clusters between $w \mathrm{Mel}$ and the most distantly related sequenced supergroup D Wolbachia genome, $w \mathrm{Bm}$, as described in the Rickettsiales Comparative Database (Dunning Hotopp et al., 2006b). The similarity of these numbers is somewhat surprising considering the similarity of the five strains compared by mCGH when compared to the divergence of $w \mathrm{Bm}$. In addition, the microarray-defined core genome uses highstringency DNA identity as opposed to the bi-directional reciprocal best protein matches for the orthologue prediction. Despite these differences, $597 \mathrm{wMel}$ proteins are present in both the orthologue prediction and the microarray-defined core genome. The convergence of these two core genome metrics across diverse strains may suggest a single Wolbachia core genome that spans the supergroup designations, thus supporting the single species designation of W. pipientis (Lo et al., 2007).

\section{Mobile genetic elements}

Bacteriophage and other mobile elements are notable catalysts of lateral gene transfer and traditionally have been considered absent in the genomes of obligate intracellular bacteria. However, in species that experience horizontal transmission to new hosts, moderate to high levels of mobile genetic elements have been observed (Bordenstein \& Reznikoff, 2005; Wu et al., 2004). Previous studies of Wolbachia phage indicate rampant lateral phage transfer between divergent strains and that the mechanism of transfer is in part mediated via the high rate of Wolbachia co-infections (Bordenstein \& Wernegreen, 2004; Masui et al., 2000). Mobility of Wolbachia phage could promote the introduction of novel genes by shuttling large fragments of Wolbachia DNA between genomes.

The mCGH results show that phage genes are significantly over-represented in the absent/divergent genes (Table 2), supporting the idea that these strains with similar genomic backbones are actively exchanging phage regions. Of the absent/divergent genes identified, $21-87 \%$ are in the prophage regions (Fig. 3). Although all of the strains examined here have portions of each prophage (Fig. 4B), each also appears to be missing sections of these regions.

One region of interest is the region encompassing WD_0612-WD_0618 that is flanked by portions of WOB. It encodes an NAD-dependent epimerase/dehydratase family protein, a glycosyltransferase with a radical SAM domain, two putative L-allo-threonine aldolases, an $\mathrm{ABC}$ transporter permease and ATP-binding protein, a conserved domain protein, and a conserved hypothetical protein (Fig. 4A). This region was conserved in all but wInn in the current study (Fig. 4B), but was not identified 

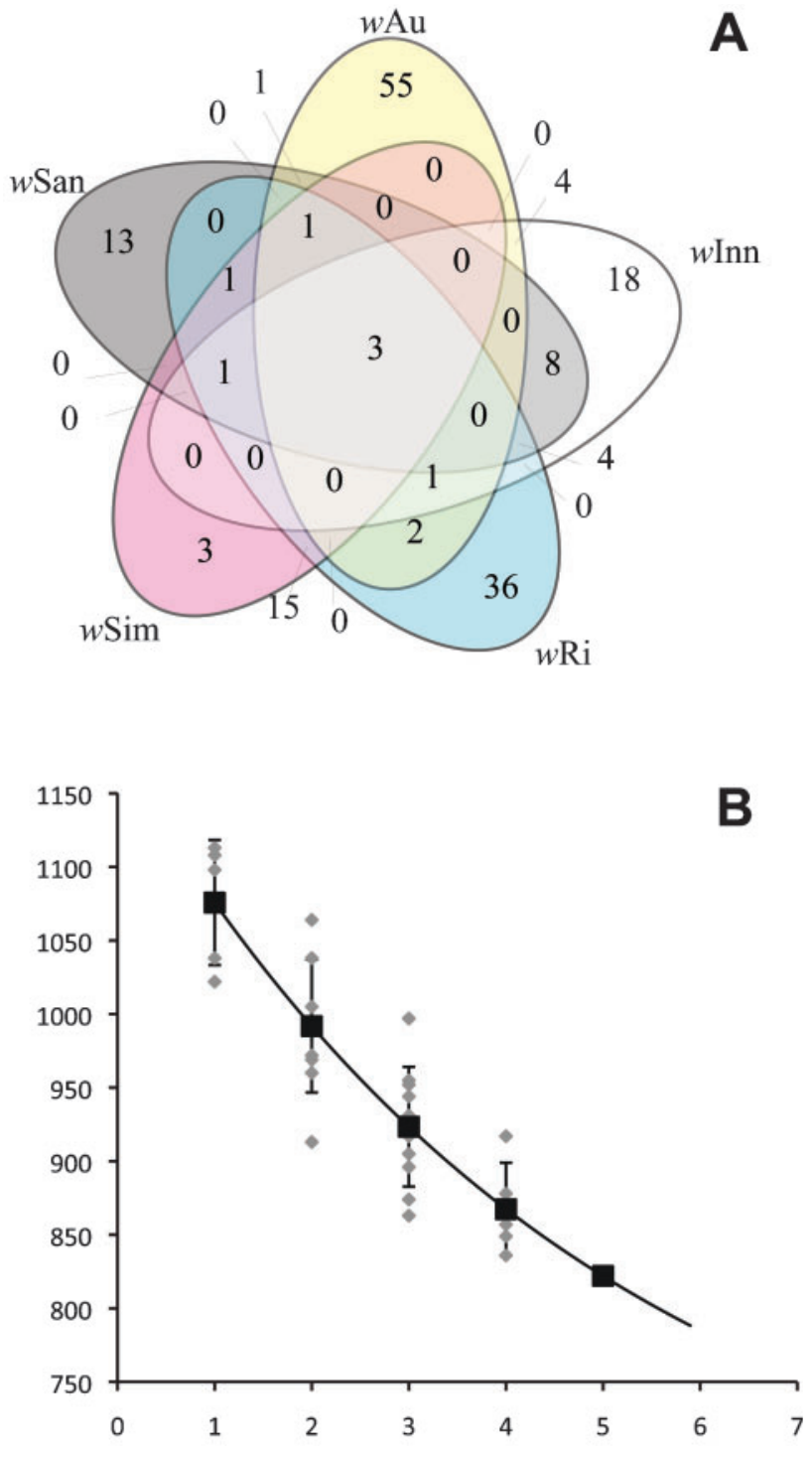

Fig. 2. Genomic diversity and the core genome. (A) Most gene differences are unique to a strain, resulting in different sets of absent/divergent genes (ratios $\geqslant 3$ ) per strain, with only three genes absent/divergent in all five strains. Numbers within the intersections of different ovals count the number of absent/ divergent genes (ratio $\geqslant 3$ ) shared by $2,3,4$ or 5 organisms. (B) The number of Wolbachia core genes for $n$ query genomes is plotted against the number of query genomes compared. For example, when $n=3$, each query genome is compared to all possible combinations of two other query genomes to see how many genes are shared between the three. Each combination $(\checkmark)$, the mean of each combination ( $\boldsymbol{\square})$, and the standard deviation (error bars) are presented. With the addition of each genome a significant number of genes are removed from the core genome, demonstrating that even between these closely related Wolbachia strains there is a sizeable amount of genomic diversity. The nonlinear regression of $620.9+557.9 \mathrm{e}^{-0.204 x} \quad(\mathrm{RSS}=0.0148)$ suggests that the core genome consists of 621 genes.
Table 2. TIGR role categories of overrepresented absent/ divergent genes

Fisher's Exact test, Bonferroni stepdown correction; $P<0.01$.

\begin{tabular}{|ll|}
\hline Strain & TIGR role category $(\boldsymbol{P}$ value $)$ \\
\hline Drosophila simulans wAu & Hypothetical proteins $\left(1.3 \times 10^{-17}\right)$ \\
Drosophila innubila $w \mathrm{Inn}$ & Hypothetical proteins $\left(8.0 \times 10^{-3}\right)$ \\
Drosophila simulans $w \mathrm{Ri}$ & Hypothetical proteins $\left(5.2 \times 10^{-6}\right)$ \\
& Prophage functions $\left(5.6 \times 10^{-4}\right)$ \\
Drosophila simulans $w$ Sim & Prophage functions $\left(9.3 \times 10^{-3}\right)$ \\
Drosophila simulans $w$ San & Prophage functions $\left(2.7 \times 10^{-10}\right)$ \\
Combined (all five strains) & Hypothetical proteins $\left(3.9 \times 10^{-26}\right)$ \\
& Prophage functions $\left(1.0 \times 10^{-4}\right)$ \\
\hline
\end{tabular}

in either the $w \mathrm{Bm}$ or $w$ Pip genome sequences. Interestingly, this same region was uncovered in a bacterial plasmid in the genome sequencing of $I$. scapularis (GenBank ABJB000000000) and is attributed to a concomitantly sequenced ovary-associated Rickettsia endosymbiont (Noda et al., 1997; Steiner et al., 2008). This endosymbiont is most closely related to other tickassociated Rickettsia spp. (Weinert et al., 2009). The plasmid is not thought to arise from a lower-level contaminating Wolbachia infection because (a) only 44 Wolbachia sequences limited to this $\sim 10 \mathrm{kbp}$ region were recovered in the whole-genome shotgun sequencing project (Dunning Hotopp et al., 2007) as opposed to the $100000+$ reads recovered with homology to the rickettsial endosymbiont; (b) the 16S rRNA and wsp genes could not be amplified from the I. scapularis genomic DNA with Wolbachia-specific primers; and (c) of the 40 nontransposase ORFs on the plasmid, 23 have a rickettsial best match (e-value $<10^{-15}$ ), including genes involved in plasmid maintenance, while only the seven corresponding to WD_0612-WD_0618 have a Wolbachia best match (e-value $<10^{-100}$ ). Of the remaining non-transposase ORFs on the plasmid, eight have a Lawsonia intracellularis best match (e-value $<10^{-50}$ ), and two have a best match to other bacteria.

In the Rickettsia endosymbiont plasmid, this $\sim 10 \mathrm{kbp}$ region is flanked by transposases (Fig. 4A). The lack of divergence ( $70 \%$ nt identity) and association with mobile elements strongly suggests lateral gene transfer between these micro-organisms. No closely related orthologues are in public databases to facilitate assigning the direction of this transfer or determining whether a third, intermediate organism was involved. Regardless, this is a compelling case of lateral gene transfer between intracellular bacteria of different genera.

As an additional example of mobile element transfer between intracellular bacteria of diverse genera, Wolbachia and the unrelated bacterial agent of cat-scratch disease, Bartonella henselae, share divergent prophage homologues of prophage WO-B (Alsmark et al., 2004). These two intracellular parasites likely exchanged phage genes in the 


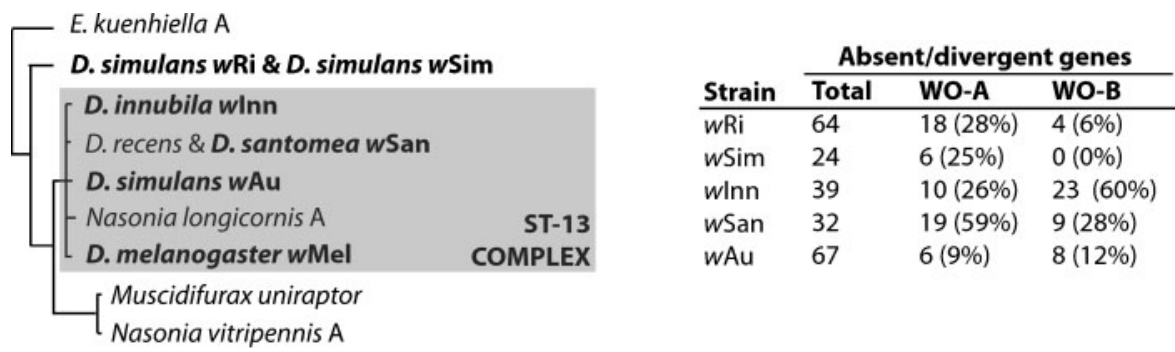

Fig. 3. MLST-based phylogeny of $m$ CGH strains. The phylogenic relationship is shown of the mCGH strains (bold) based on MLST (figure adapted from Baldo et al., 2006b). The ST-13 complex is boxed in grey. This tree is contrasted to the total number of absent/divergent genes and the absent/divergent genes in the vicinity of prophages WO-A and WO-B. Since exact prophage boundaries cannot be discerned, for this analysis WO-A and WO-B are defined as the regions from WD_0250 to WD_0300 and WD_0581 to WD_0650, respectively. Large portions of the absent/divergent genes are found in the vicinity of the prophage. Despite close relationships among strains based on MLST, mCGH reveals significant levels of genome divergence among them.

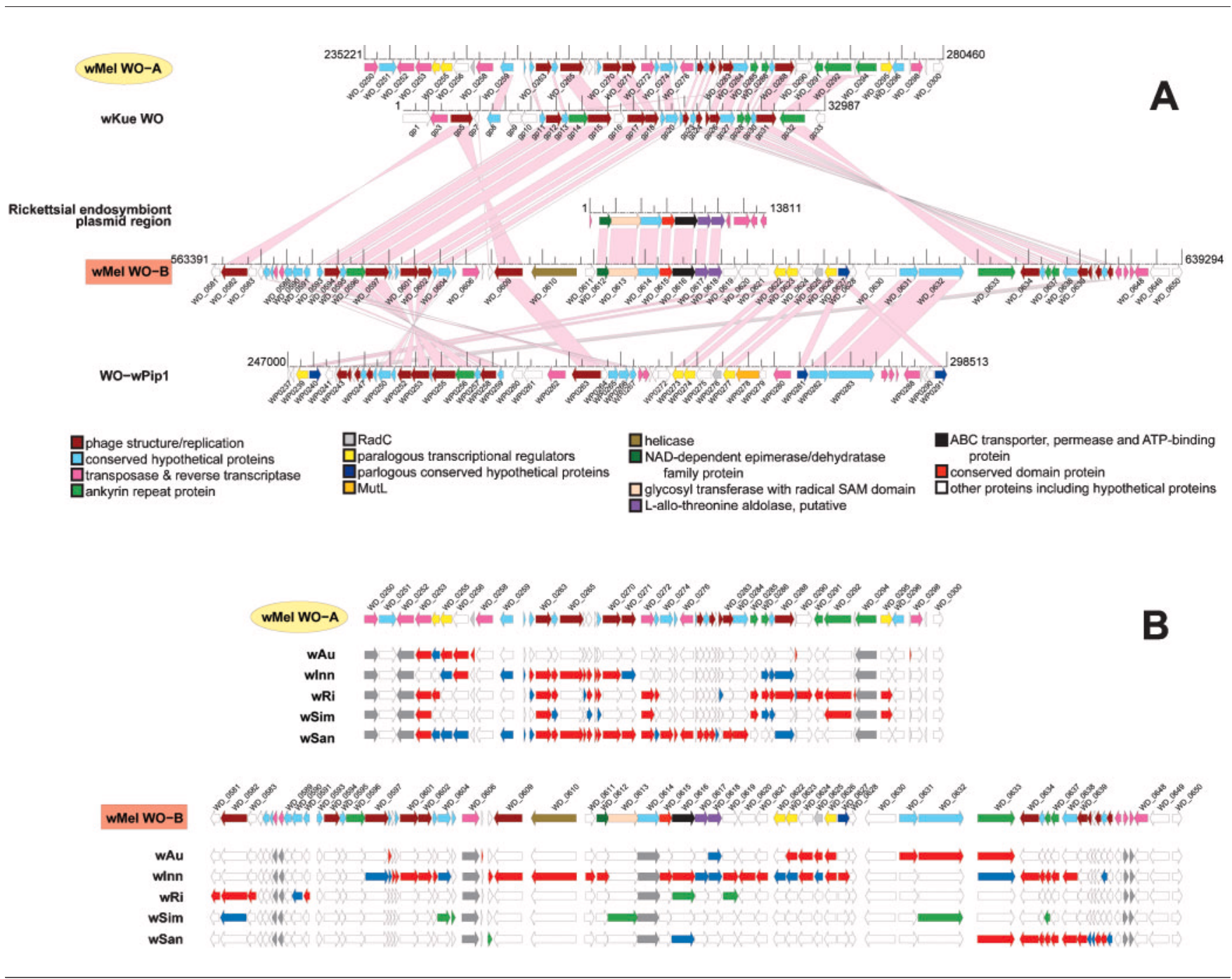

Fig. 4. Prophage regions. Four prophage regions are compared, including the canonical $w K$ Kue WO phage, the two $w M e l$ WO prophage, and a representative prophage from $w P i p(A)$. Absent/divergent genes (red; ratio $>3$ ), ambiguous genes (blue; ratio 23 ) and possible duplicated genes (green; ratio $<0.3$ ) are shown under the representative $w$ Mel prophage region $(B)$. The prophage regions appear to have a high level of variation. A region flanked by wMel's WO-B is homologous to a plasmid sequenced from a rickettsial endosymbiont of $I$. scapularis (A). This region appears to be the result of lateral gene transfer between Wolbachia and Rickettsia. It is present in wAu, wSim, wRi and $w$ San in the current study, but not in the genomes of $w B m$ or $w P i p(B)$. 
evolutionary past within their common invertebrate host, the cat flea Ctenophalides felis (Casiraghi et al., 2005; Rolain et al., 2003).

\section{Ankyrin repeat proteins and confirmation of mCGH results}

The sequencing and annotation of the $w \mathrm{Mel}$ genome revealed 23 genes encoding ankyrin domain repeats, one of the highest levels in a prokaryotic genome $(\mathrm{Wu}$ et al., 2004). The functions of these proteins in $w \mathrm{Mel}$ are not known, although ankyrin repeats often promote proteinprotein interactions. In eukaryotes, they can be involved in many functions, including cell cycle regulation and cytoskeleton interactions (Voronin \& Kiseleva, 2007; Wu et al., 2004), making them candidates for mediating Wolbachia-host interaction and host reproductive phenotypes (Foster et al., 2005; Iturbe-Ormaetxe et al., 2005a). In other symbiotic relationships, ankyrin repeat genes have been shown to bind to host chromatin in A. phagocytophilum (Caturegli et al., 2000; Park et al., 2004), have a role in hydrogen peroxide resistance of Pseudomonas aeruginosa (Howell et al., 2000) and be key in establishing a plantbacterium relationship between Lotus japonicus and Mesorhizobium loti (Kumagai et al., 2007).

The ankyrin protein content of nine Wolbachia strains was determined previously using hybridization and PCR amplification (Iturbe-Ormaetxe et al., 2005a). A comparison of the two strains overlapping with this current study reveals a high degree of agreement. Using a ratio cutoff of 3 and with the exception of WD_0633, the results for strain $w \mathrm{Au}$ are in agreement with those previous results, revealing that all of the examined ankyrin genes from $w \mathrm{Mel}$ are present in $w \mathrm{Au}$. In strain $w \mathrm{Ri}$, three genes (WD_0566, WD_0147, WD_0754) were previously demonstrated as being present in $w \mathrm{Ri}$ but are suggested to be absent/ divergent in the current mCGH study. There are several possible explanations for these differences, including: (1) size and sequence of the hybridization probe/target pairs in the two studies, (2) sensitivity, particularly pertaining to single- versus dual-channel hybridization experiments, (3) cutoff criteria for assessing background, (4) data normalization, (5) hybridization stringency, or (6) polymorphic populations. However, despite these differences there is a high degree of concordance in the dataset.

In the current study, only $w \mathrm{Ri}$ and $w$ Sim had an overrepresentation of absent/divergent ankyrin genes (Fisher's exact text, $P<0.01)$. Phage activity, along with the movement of transposable genetic elements, may help to explain the marked changes in the ankyrin gene content, as most absent/divergent ankyrin repeat genes were localized to the prophage WO-A in these two genomes. However, this does not reflect an absence of the prophage, as WO-A structural genes and some conserved hypothetical genes are conserved (Fig. 4B). In contrast, wSan contains the ankyrin repeat proteins in $\mathrm{WO}-\mathrm{A}$, but has missing/divergent phage components (Fig. 4B). Instead of prophage absence, this likely reflects either differential loss of portions of the prophage region after incorporation into the genome and/ or independent acquisition of the ankyrin genes. There appears to be a similar, but lower-level, variation in the WO-B ankyrin repeat genes in wSan and wInn (Fig. 4B), suggesting the presence of related prophage regions in Wolbachia strains with differing content of ankyrin repeat proteins.

\section{Hypothetical proteins}

Hypothetical proteins are also overrepresented in the absent/divergent genes in this study (Table 2). There are 72 hypothetical proteins ranging in size from 30 to 2843 aa with a ratio $>3$, suggesting absence/divergence in at least one strain. All of these proteins were re-examined in order to improve annotation. There are $19(26 \%)$ that are conserved with $w$ Pip, but 66 (92\%) remain hypothetical proteins unique to arthropod Wolbachia. Of the remaining six genes, three have orthologues in the $W$. pipientis $w \mathrm{Bm}$ genome (WD_0424, WD_0434, WD_1039), one has an orthologue in the Rickettsia felis genome (WD_0268), one has an orthologue in Ehrlichia chaffeensis (WD_0971), and one has orthologues in Anopheles gambiae and Aedes aegypti (WD_0513). A third of the absent/divergent hypothetical proteins are $>150$ aa, while the mean and median sizes are 209 aa and 68 aa, respectively. By comparison, the hypothetical proteins in the entire genome have the same size range but with mean and median sizes of 180 aa and 95 aa, respectively. Therefore, it is not merely the smallest hypothetical proteins, which one might hypothesize are incorrectly predicted to encode proteins, that are absent/divergent. As the unique genes in the genome, those encoding hypothetical proteins may reflect the genetic diversity of Wolbachia.

\section{Divergence of strains}

Most gene differences were unique to a strain, resulting in a unique set of absent/divergent genes per strain (Fig. 2A). Only three genes were present in $w \mathrm{Mel}$ but absent/ divergent in all other strains examined. These adjacent genes include a DNA mismatch repair protein MutL-2 (WD_0509), a degenerate RNase (WD_0510), and a conserved hypothetical protein (WD_0511). The region from WD_0509 to WD_0514 was previously hypothesized to be horizontally transferred into $w$ Mel based on absence in the Wolbachia draft genomes of $w$ Ana and wSim (Salzberg et al., 2005). In the current study, WD_0512 and WD_0513 are also absent/divergent in wAu and showed higher than average ratios in all strains but $w$ Sim. Using PCR and Southern hybridization, WD_0509-WD_0514 were found to be absent in $w \mathrm{Au}$ and $w \mathrm{Ri}$ while only partially absent in wSan, confirming the mCGH studies. In wSim, these WD_0512 and WD_0513 genes actually showed lower than average ratios, suggesting multiple copies. However, these regions are absent in the $D$. simulans genome sequencing project and corresponding 
wSim Wolbachia draft genome (Salzberg et al., 2005). These discrepancies may be attributed to a cloning bias in the whole-genome shotgun sequencing project or a Wolbachia population polymorphic for this region. Although unusual, a polymorphic population has some precedent. In the sequencing of $w$ Pip, the laboratory, inbred mosquito line used was not clonal, leading to the suggestion that Wolbachia can rapidly generate chromosomal variation (Klasson et al., 2008).

WD_0513 is interesting as its closest putative homologues are found in the genomes of Anopheles gambiae, Aedes aegypti and Culex quinquefasciatus (Woolfit et al., 2009) and facilitate invasion of malarial sporozoites into salivary gland cells (Arca et al., 2005; Korochkina et al., 2006). Prior analyses revealed that the co-transcribed WD_0512WD_0514 genes (Iturbe-Ormaetxe et al., 2005a) are frequently absent from Wolbachia genomes with the exception of $w$ Pip (Korochkina et al., 2006) and Wolbachia closely related to $w \mathrm{Mel}$ including $w \mathrm{MelCS}$ and $w$ MelPop (Iturbe-Ormaetxe et al., 2005a). The limited distribution of these proteins in only mosquitoes and select Wolbachia strains suggests host/endosymbiont lateral gene transfer. Lateral transfer of Wolbachia genes to animal hosts has been described (Dunning Hotopp et al., 2007; Kondo et al., 2002), but in the case of WD_0513, the actual direction of this transfer is difficult to ascertain without further knowledge of the gene's phylogenetic distribution (Woolfit et al., 2009). However, the limited distribution of this region and the presence of these proteins in both the endosymbiont and host make these genes interesting candidates for research into host-parasite interactions.

Although all of the strains examined here had distinct genomic content, $w \mathrm{Ri}$ and $w$ Sim were found to be most similar (Figs 2A, 3 and 5). It has been hypothesized that

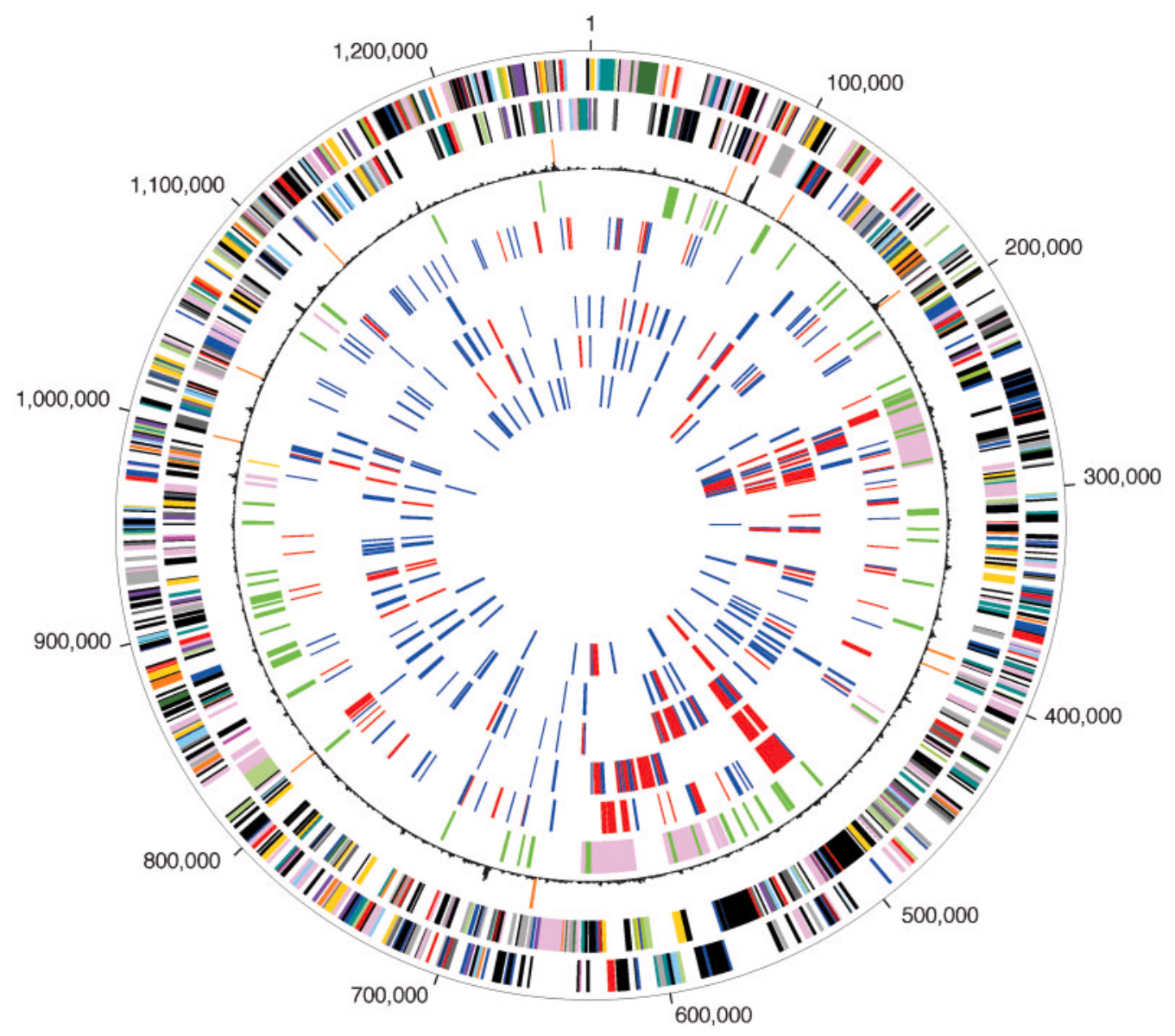

Fig. 5. Circular representation of $\mathrm{mCGH}$ results. The outer two rims present the predicted coding regions on the plus and minus strand, respectively, colour-coded by role categories. Rim 3 contains the genes not represented on the array (orange) and atypical nucleotide content (black). Rim 4 indicates the origin of replication (gold), phage genes (pink), and transposon genes (green). Rims 5-9 are the comparative genome hybridization results for $w A \mathrm{u}, w \mathrm{lnn}, w \mathrm{Ri}, w S i m$ and $w S a n$, respectively. Using previously determined cutoffs, the following assignments were made: a ratio $>3$ is most likely absent or divergent in the test strain (red); a ratio between 2 and 3 is absence/divergence not predicted (blue); a ratio $<2$ is most likely present in the test strain (white). Considerable diversity is present in the absent/divergent genes, particularly in the vicinity of phage (rim 4, pink). 
these are actually the same strain (Iturbe-Ormaetxe et al., 2005b), and the two strains are identical in their MLST alleles (Fig. 3). In this current study, the divergence of strains could not be determined using microarray support trees analysis as all nodes had $<50 \%$ support ( TMeV support trees, average linkage, Euclidean distance, bootstrap 100 iterations; data not shown). However, these strains had the highest number of absent/divergent genes in common in all of the two-way comparisons (Fig. 2A). Despite this commonality, there are 46 differences in absent/divergent genes between the two strains while only $21 \mathrm{absent} /$ divergent genes overlapped in these two strains (Fig. 2A), suggesting distinct genomic content.

\section{DISCUSSION}

\section{Genome diversity}

Although Wolbachia are obligate intracellular bacteria, they are considered facultative endosymbionts because hosts can survive uninfected. They are cosmopolitan, with host promiscuity and frequent co-infection with other Wolbachia strains and probably other intracellular bacteria. These factors combined likely increase the opportunity of Wolbachia to exchange genomic DNA. This increased genomic exchange may promote genetic and phenotypic diversity and contribute to Wolbachia's success as one of the most abundant intracellular bacteria.

\section{Lateral gene transfer}

In the supergroup A and B Wolbachia, intraspecies lateral gene transfer is facilitated by prevalent infections (Hilgenboecker et al., 2008), transfer of mobile elements (Bordenstein \& Wernegreen, 2004; Masui et al., 2000) and extensive recombination (Baldo et al., 2006a, b; Lo et al., 2007; Verne et al., 2007). It has not been clear if Wolbachia can exchange DNA through lateral gene transfer with other bacteria. In order for this to happen, Wolbachia must come into contact with other bacteria either inside the cell or as it is passing between cells/organisms (e.g. in the haemolymph). We show here a region shared at $\sim 70 \%$ nucleotide identity flanked by transposases on a plasmid in a Rickettsia endosymbiont and flanked by prophage sequences in a Wolbachia. The direction of the transfer is difficult to identify. This region is not found in all Rickettsia spp. or all Wolbachia strains, and has no close similarity to other organisms to allow a phylogenetic assignment. Both organisms can infect ovaries and can infect I. scapularis (Benson et al., 2004), possibly providing the opportunity for plasmid transfer. Alternatively, another host or a third DNA reservoir may be involved in mediating this transfer.

\section{Limitations/benefits of $\mathrm{mCGH}$}

mCGH is of limited use in genomic comparison of intracellular bacteria with low genomic diversity. For example, a comparison of two A. phagocytophilum genomes revealed that only four $p 44$ genes varied between the strains (Dunning Hotopp et al., 2006b). These encompass a subset of genes that were already known to be highly variable. But in the case of Wolbachia, where large genomic variation is seen, mCGH is more fruitful.

The mCGH platform may become obsolete as sequencing costs fall, but with in-house printed arrays the cost is still an order of magnitude less than most commercial microarray platforms and two orders of magnitude less than data obtained through next-generation sequencing. So mCGH may still have significant use in assessing genomic diversity, particularly before selecting strains to be sequenced.

One problem with examining obligate intracellular bacteria with any genomic platform is acquiring microgram quantities of DNA that are devoid of host genomic DNA contamination. Better methods are needed to obtain purified material and to amplify materials when genomic DNA quantity is limited. In this current study, several strains had to be eliminated due to high levels of background hybridization, likely owing to host DNA contamination. Likewise, because of limiting quantities of highly purified Wolbachia DNA, whole-genome amplification could not be avoided. These limiting DNA quantities precluded assessment of bias in the current study, but the amplification results were shown to be reproducible. By pooling multiple amplification reactions, performing multiple replicate hybridizations, and taking the median value of those replicates any biases should be minimized.

One of the limitations of mCGH, when compared to sequencing, is that it only assesses gene content represented on the microarray. As such, only sequences represented in public databases are queried. Although great genomic diversity of $w \mathrm{Mel}$ prophage regions is demonstrated, novel phage regions and genes cannot be assessed using the mCGH technique. The high levels of variation in these regions suggest that further sequencing of Wolbachia and its phage would be particularly fruitful.

\section{Concluding remarks}

In Wolbachia, mCGH can identify variable regions including phage regions, ankyrin repeat proteins, and hypothetical proteins. However, one of the limitations is the ability to only characterize the diversity relative to known gene content. The unique genes in each strain examined remain elusive. But even with this limitation, the difference in gene content is the greatest variation observed for closely related obligate intracellular bacteria, with Wolbachia phages being a major source of variation. Wolbachia sequencing has been stymied due to the high levels of host DNA contamination. But as the costs of genome sequencing decrease and new sequencing technologies are developed that alleviate this burden, Wolbachia strains should be targeted for further sequencing 
to better understand their uniquely high levels of genetic diversity among endosymbionts.

\section{ACKNOWLEDGEMENTS}

This work was supported by funds from US National Science Foundation grant EF-0328363 to J. Werren, by funds from EU CSAREGPROT 203590 - MicrobeGR and intramural funds of the University of Ioannina to K. B., by funds from US National Science Foundation grant IOS-0852344 and US National Institutes of Health grant RO1-GM085163-01 to S. B. Author contributions: Conceived and designed experiments: Seth Bordenstein, Kostas Bourtzis, Julie C. Dunning Hotopp, Hervé Tettelin, John Werren. Experimental work: Sarah Biber, Seth Bordenstein, Julie C. Dunning Hotopp, Panos Ioannidis, Nadeeza Ishmael, Stefanos Siozios. Data analysis: Seth Bordenstein, Kostas Bourtzis, Julie C. Dunning Hotopp, Panos Ioannidis, Nadeeza Ishmael, Joyce Sakamoto, Hervé Tettelin, John Werren. Contributed reagents/materials/analysis tools: Vish Nene. Manuscript writing and editing: Seth Bordenstein, Kostas Bourtzis, Julie C. Dunning Hotopp, Nadeeza Ishmael, Hervé Tettelin, John Werren.

\section{REFERENCES}

Alsmark, C. M., Frank, A. C., Karlberg, E. O., Legault, B. A., Ardell, D. H., Canbäck, B., Eriksson, A. S., Näslund, A. K., Handley, S. A. \& other authors (2004). The louse-borne human pathogen Bartonella quintana is a genomic derivative of the zoonotic agent Bartonella henselae. Proc Natl Acad Sci U S A 101, 9716-9721.

Arca, B., Lombardo, F., Valenzuela, J. G., Francischetti, I. M., Marinotti, O., Coluzzi, M. \& Ribeiro, J. M. (2005). An updated catalogue of salivary gland transcripts in the adult female mosquito, Anopheles gambiae. J Exp Biol 208, 3971-3986.

Baldo, L., Bordenstein, S., Wernegreen, J. J. \& Werren, J. H. (2006a). Widespread recombination throughout Wolbachia genomes. Mol Biol Evol 23, 437-449.

Baldo, L., Dunning Hotopp, J. C., Jolley, K. A., Bordenstein, S. R., Biber, S. A., Choudhury, R. R., Hayashi, C., Maiden, M. C., Tettelin, H. \& Werren, J. H. (2006b). Multilocus sequence typing system for the endosymbiont Wolbachia pipientis. Appl Environ Microbiol 72, 70987110.

Behr, M. A., Wilson, M. A., Gill, W. P., Salamon, H., Schoolnik, G. K., Rane, S. \& Small, P. M. (1999). Comparative genomics of BCG vaccines by whole-genome DNA microarray. Science 284, 1520-1523.

Benson, M. J., Gawronski, J. D., Eveleigh, D. E. \& Benson, D. R. (2004). Intracellular symbionts and other bacteria associated with deer ticks (Ixodes scapularis) from Nantucket and Wellfleet, Cape Cod, Massachusetts. Appl Environ Microbiol 70, 616-620.

Bordenstein, S. R. \& Reznikoff, W. S. (2005). Mobile DNA in obligate intracellular bacteria. Nat Rev Microbiol 3, 688-699.

Bordenstein, S. \& Rosengaus, R. B. (2005). Discovery of a novel Wolbachia supergroup in Isoptera. Curr Microbiol 51, 393-398.

Bordenstein, S. R. \& Wernegreen, J. J. (2004). Bacteriophage flux in endosymbionts (Wolbachia): infection frequency, lateral transfer, and recombination rates. Mol Biol Evol 21, 1981-1991.

Bordenstein, S. R., Paraskevopoulos, C., Dunning Hotopp, J. C., Sapountzis, P., Lo, N., Bandi, C., Tettelin, H., Werren, J. H. \& Bourtzis, K. (2008). Parasitism and mutualism in Wolbachia: what the phylogenomic trees can and can not say. Mol Biol Evol 26, 231-241.

Boucher, Y., Nesbo, C. L. \& Doolittle, W. F. (2001). Microbial genomes: dealing with diversity. Curr Opin Microbiol 4, 285-289.
Bouchon, D., Rigaud, T. \& Juchault, P. (1998). Evidence for widespread Wolbachia infection in isopod crustaceans: molecular identification and host feminization. Proc Biol Sci 265, 1081-1090.

Casiraghi, M., Favia, G., Cancrini, G., Bartoloni, A. \& Bandi, C. (2001). Molecular identification of Wolbachia from the filarial nematode Mansonella ozzardi. Parasitol Res 87, 417-420.

Casiraghi, M., Bain, O., Guerrero, R., Martin, C., Pocacqua, V., Gardner, S. L., Franceschi, A. \& Bandi, C. (2004). Mapping the presence of Wolbachia pipientis on the phylogeny of filarial nematodes: evidence for symbiont loss during evolution. Int $J$ Parasitol 34, 191-203.

Casiraghi, M., Bordenstein, S. R., Baldo, L., Lo, N., Beninati, T., Wernegreen, J. J., Werren, J. H. \& Bandi, C. (2005). Phylogeny of Wolbachia pipientis based on gltA, groEL and fts $Z$ gene sequences: clustering of arthropod and nematode symbionts in the F supergroup, and evidence for further diversity in the Wolbachia tree. Microbiology 151, 4015-4022.

Caturegli, P., Asanovich, K. M., Walls, J. J., Bakken, J. S., Madigan, J. E., Popov, V. L. \& Dumler, J. S. (2000). ankA: an Ehrlichia phagocytophila group gene encoding a cytoplasmic protein antigen with ankyrin repeats. Infect Immun 68, 5277-5283.

Czarnetzki, A. B. \& Tebbe, C. C. (2004). Detection and phylogenetic analysis of Wolbachia in Collembola. Environ Microbiol 6, 35-44.

Dobson, S. L., Marsland, E. J. \& Rattanadechakul, W. (2002). Mutualistic Wolbachia infection in Aedes albopictus: accelerating cytoplasmic drive. Genetics 160, 1087-1094.

Dumler, J. S., Barbet, A. F., Bekker, C. P., Dasch, G. A., Palmer, G. H., Ray, S. C., Rikihisa, Y. \& Rurangirwa, F. R. (2001). Reorganization of genera in the families Rickettsiaceae and Anaplasmataceae in the order Rickettsiales: unification of some species of Ehrlichia with Anaplasma, Cowdria with Ehrlichia and Ehrlichia with Neorickettsia, descriptions of six new species combinations and designation of Ehrlichia equi and 'HGE agent' as subjective synonyms of Ehrlichia phagocytophila. Int J Syst Evol Microbiol 51, 2145-2165.

Dunning Hotopp, J. C., Grifantini, R., Kumar, N., Tzeng, Y. L., Fouts, D., Frigimelica, E., Draghi, M., Giuliani, M. M., Rappuoli, R. \& other authors (2006a). Comparative genomics of Neisseria meningitidis: core genome, islands of horizontal transfer and pathogen-specific genes. Microbiology 152, 3733-3749.

Dunning Hotopp, J. C., Lin, M., Madupu, R., Crabtree, J., Angiuoli, S. V., Eisen, J. A., Seshadri, R., Ren, Q., Wu, M. \& other authors (2006b). Comparative genomics of emerging human ehrlichiosis agents. PLoS Genet 2, e21.

Dunning Hotopp, J. C., Clark, M. E., Oliveira, D. C., Foster, J. M., Fischer, P., Torres, M. C., Giebel, J. D., Kumar, N., Ishmael, N. \& other authors (2007). Widespread lateral gene transfer from intracellular bacteria to multicellular eukaryotes. Science 317, 1753-1756.

Dyer, K. A. \& Jaenike, J. (2005). Evolutionary dynamics of a spatially structured host-parasite association: Drosophila innubila and malekilling Wolbachia. Evolution 59, 1518-1528.

Foster, J., Ganatra, M., Kamal, I., Ware, J., Makarova, K., Ivanova, N., Bhattacharyya, A., Kapatral, V., Kumar, S. \& other authors (2005). The Wolbachia genome of Brugia malayi: endosymbiont evolution within a human pathogenic nematode. PLoS Biol 3, e121.

Heath, B. D., Butcher, R. D., Whitfield, W. G. \& Hubbard, S. F. (1999). Horizontal transfer of Wolbachia between phylogenetically distant insect species by a naturally occurring mechanism. Curr Biol 9, 313316.

Hedges, L. M., Brownlie, J. C., O’Neill, S. L. \& Johnson, K. N. (2008). Wolbachia and virus protection in insects. Science 322, 702.

Hilgenboecker, K., Hammerstein, P., Schlattmann, P., Telschow, A. \& Werren, J. H. (2008). How many species are infected with 
Wolbachia? - A statistical analysis of current data. FEMS Microbiol Lett 281, 215-220.

Hoffmann, A. A., Turelli, M. \& Simmons, G. M. (1986). Unidirectional incompatibility between populations of Drosophila simulans. Evolution 40, 692-701.

Hoffmann, A. A., Clancy, D. \& Duncan, J. (1996). Naturally-occurring Wolbachia infection in Drosophila simulans that does not cause cytoplasmic incompatibility. Heredity 76, 1-8.

Hogg, J. S., Hu, F. Z., Janto, B., Boissy, R., Hayes, J., Keefe, R., Post, J. C. \& Ehrlich, G. D. (2007). Characterization and modeling of the Haemophilus influenzae core and supragenomes based on the complete genomic sequences of Rd and 12 clinical nontypeable strains. Genome Biol 8, R103.

Holden, P. R., Brookfield, J. F. \& Jones, P. (1993). Cloning and characterization of an $\mathrm{fts} Z$ homologue from a bacterial symbiont of Drosophila melanogaster. Mol Gen Genet 240, 213-220.

Howell, M. L., Alsabbagh, E., Ma, J. F., Ochsner, U. A., Klotz, M. G., Beveridge, T. J., Blumenthal, K. M., Niederhoffer, E. C., Morris, R. E. $\&$ other authors (2000). AnkB, a periplasmic ankyrin-like protein in Pseudomonas aeruginosa, is required for optimal catalase B (KatB) activity and resistance to hydrogen peroxide. J Bacteriol 182, 45454556.

Huigens, M. E., de Almeida, R. P., Boons, P. A., Luck, R. F. \& Stouthamer, R. (2004). Natural interspecific and intraspecific horizontal transfer of parthenogenesis-inducing Wolbachia in Trichogramma wasps. Proc Biol Sci 271, 509-515.

Iturbe-Ormaetxe, I., Burke, G. R., Riegler, M. \& O'Neill, S. L. (2005a). Distribution, expression, and motif variability of ankyrin domain genes in Wolbachia pipientis. J Bacteriol 187, 5136-5145.

Iturbe-Ormaetxe, I., Riegler, M. \& O'Neill, S. L. (2005b). New names for old strains? Wolbachia wSim is actually wRi. Genome Biol 6, 401 (author reply 401).

Klasson, L., Walker, T., Sebaihia, M., Sanders, M. J., Quail, M. A., Lord, A., Sanders, S., Earl, J., O'Neill, S. L. \& other authors (2008). Genome evolution of Wolbachia strain $w$ Pip from the Culex pipiens group. Mol Biol Evol 25, 1877-1887.

Klasson, L., Westberg, J., Sapountzis, P., Näslund, K., Lutnaes, Y., Darby, A. C., Veneti, Z., Chen, L., Braig, H. R. \& other authors (2009). The mosaic genome structure of the Wolbachia $w \mathrm{Ri}$ strain infecting Drosophila simulans. Proc Natl Acad Sci U S A 106, 5725-5730.

Kondo, N., Nikoh, N., Ijichi, N., Shimada, M. \& Fukatsu, T. (2002). Genome fragment of Wolbachia endosymbiont transferred to $\mathrm{X}$ chromosome of host insect. Proc Natl Acad Sci U S A 99, 1428014285 .

Korochkina, S., Barreau, C., Pradel, G., Jeffery, E., Li, J., Natarajan, R. Shabanowitz, J., Hunt, D., Frevert, U. \& Vernick, K. D. (2006). A mosquito-specific protein family includes candidate receptors for malaria sporozoite invasion of salivary glands. Cell Microbiol 8, 163175.

Kumagai, H., Hakoyama, T., Umehara, Y., Sato, S., Kaneko, T., Tabata, S. \& Kouchi, H. (2007). A novel ankyrin-repeat membrane protein, IGN1, is required for persistence of nitrogen-fixing symbiosis in root nodules of Lotus japonicus. Plant Physiol 143, 1293-1305.

Lan, R. \& Reeves, P. R. (2000). Intraspecies variation in bacterial genomes: the need for a species genome concept. Trends Microbiol 8, 396-401.

Lo, N., Casiraghi, M., Salati, E., Bazzocchi, C. \& Bandi, C. (2002). How many Wolbachia supergroups exist? Mol Biol Evol 19, 341-346.

Lo, N., Paraskevopoulos, C., Bourtzis, K., O'Neill, S. L., Werren, J. H., Bordenstein, S. R. \& Bandi, C. (2007). Taxonomic status of the intracellular bacterium Wolbachia pipientis. Int J Syst Evol Microbiol 57, 654-657.
Masui, S., Kamoda, S., Sasaki, T. \& Ishikawa, H. (2000). Distribution and evolution of bacteriophage WO in Wolbachia, the endosymbiont causing sexual alterations in arthropods. J Mol Evol 51, 491-497.

Medini, D., Donati, C., Tettelin, H., Masignani, V. \& Rappuoli, R. (2005). The microbial pan-genome. Curr Opin Genet Dev 15, 589594.

Noda, H., Munderloh, U. G. \& Kurtti, T. J. (1997). Endosymbionts of ticks and their relationship to Wolbachia spp. and tick-borne pathogens of humans and animals. Appl Environ Microbiol 63, 3926-3932.

O'Neill, S. L., Giordano, R., Colbert, A. M., Karr, T. L. \& Robertson, H. M. (1992). 16S rRNA phylogenetic analysis of the bacterial endosymbionts associated with cytoplasmic incompatibility in insects. Proc Natl Acad Sci U S A 89, 2699-2702.

Park, J., Kim, K. J., Choi, K. S., Grab, D. J. \& Dumler, J. S. (2004). Anaplasma phagocytophilum AnkA binds to granulocyte DNA and nuclear proteins. Cell Microbiol 6, 743-751.

Rasgon, J. L. \& Scott, T. W. (2004). Phylogenetic characterization of Wolbachia symbionts infecting Cimex lectularius L. and Oeciacus vicarius Horvath (Hemiptera: Cimicidae). J Med Entomol 41, 11751178.

Rolain, J. M., Franc, M., Davoust, B. \& Raoult, D. (2003). Molecular detection of Bartonella quintana, B. koehlerae, B. henselae, B. clarridgeiae, Rickettsia felis, and Wolbachia pipientis in cat fleas, France. Emerg Infect Dis 9, 338-342.

Salzberg, S. L., Dunning Hotopp, J. C., Delcher, A. L., Pop, M., Smith, D. R., Eisen, M. B. \& Nelson, W. C. (2005). Serendipitous discovery of Wolbachia genomes in multiple Drosophila species. Genome Biol 6, R23.

Sambrook, J., Firitsch, E. F. \& Maniatis, T. (1989). Molecular Cloning: a Laboratory Manual. Cold Spring Harbor, NY: Cold Spring Harbor Laboratory.

Steiner, F. E., Pinger, R. R., Vann, C. N., Grindle, N., Civitello, D., Clay, K. \& Fuqua, C. (2008). Infection and co-infection rates of Anaplasma phagocytophilum variants, Babesia spp., Borrelia burgdorferi, and the rickettsial endosymbiont in Ixodes scapularis (Acari: Ixodidae) from sites in Indiana, Maine, Pennsylvania, and Wisconsin. J Med Entomol 45, 289-297.

Stouthamer, R., Breeuwer, J. A. \& Hurst, G. D. (1999). Wolbachia pipientis: microbial manipulator of arthropod reproduction. Annu Rev Microbiol 53, 71-102.

Teixeria, L., Ferreira, A. \& Ashburner, M. (2008). The bacterial symbiont Wolbachia induces resistance to RNA viral infections in Drosophila melanogaster. PLoS Biol 6, e1000002.

Tettelin, H., Nelson, K. E., Paulsen, I. T., Eisen, J. A., Read, T. D., Peterson, S., Heidelberg, J., DeBoy, R. T., Haft, D. H. \& other authors (2001). Complete genome sequence of a virulent isolate of Streptococcus pneumoniae. Science 293, 498-506.

Tettelin, H., Masignani, V., Cieslewicz, M. J., Donati, C., Medini, D., Ward, N. L., Angiuoli, S. V., Crabtree, J., Jones, A. L. \& other authors (2005). Genome analysis of multiple pathogenic isolates of Streptococcus agalactiae: implications for the microbial "pangenome". Proc Natl Acad Sci U S A 102, 13950-13955.

Tettelin, H., Riley, D., Cattuto, C. \& Medini, D. (2008). Comparative genomics: the bacterial pan-genome. Curr Opin Microbiol 11, 472477.

Turelli, M. \& Hoffmann, A. A. (1995). Cytoplasmic incompatibility in Drosophila simulans: dynamics and parameter estimates from natural populations. Genetics 140, 1319-1338.

Vandekerckhove, T. T., Watteyne, S., Willems, A., Swings, J. G., Mertens, J. \& Gillis, M. (1999). Phylogenetic analysis of the $16 \mathrm{~S}$ rDNA of the cytoplasmic bacterium Wolbachia from the novel host Folsomia 
candida (Hexapoda, Collembola) and its implications for wolbachial taxonomy. FEMS Microbiol Lett 180, 279-286.

Verne, S., Johnson, M., Bouchon, D. \& Grandjean, F. (2007). Evidence for recombination between feminizing Wolbachia in the isopod genus Armadillidium. Gene 397, 58-66.

Voronin, D. A. \& Kiseleva, E. V. (2007). Functional role of proteins containing ankyrin repeats. Tsitologiia 49, 989-999.

Weinert, L. A., Werren, J. H., Aebi, A., Stone, G. N. \& Jiggins, F. M. (2009). Evolution and diversity of Rickettsia bacteria. BMC Biol 7, 6.

Wernegreen, J. J., Lazarus, A. B. \& Degnan, P. H. (2002). Small genome of Candidatus Blochmannia, the bacterial endosymbiont of Camponotus, implies irreversible specialization to an intracellular lifestyle. Microbiology 148, 2551-2556.

Werren, J. H., Zhang, W. \& Guo, L. R. (1995). Evolution and phylogeny of Wolbachia: reproductive parasites of arthropods. Proc Biol Sci 261, 55-63.

Werren, J. H., Baldo, L. \& Clark, M. E. (2008). Wolbachia: master manipulators of invertebrate biology. Nat Rev Microbiol 6, 741-751.

Woolfit, M., Iturbe-Ormaetxe, I., McGraw, E. A. \& O'Neill, S. L. (2009). An ancient horizontal gene transfer between mosquito and the endosymbiotic bacterium Wolbachia pipientis. Mol Biol Evol 26, 367-374.

Wu, M., Sun, L. V., Vamathevan, J., Riegler, M., Deboy, R., Brownlie, J. C., McGraw, E. A., Martin, W., Esser, C. \& other authors (2004). Phylogenomics of the reproductive parasite Wolbachia pipientis wMel: a streamlined genome overrun by mobile genetic elements. PLoS Biol 2, e69.

Zabalou, S., Charlat, S., Nirgianaki, A., Lachaise, D., Mercot, H. \& Bourtzis, K. (2004). Natural Wolbachia infections in the Drosophila yakuba species complex do not induce cytoplasmic incompatibility but fully rescue the $w$ Ri modification. Genetics 167, 827-834.

Zabalou, S., Apostolaki, A., Pattas, S., Veneti, Z., Paraskevopoulos, C., Livadaras, I., Markakis, G., Brissac, T., Merçot, H. \& Bourtzis, K. (2008). Multiple rescue factors within a Wolbachia strain. Genetics 178, 2145-2160.

Zeh, J. A. \& Zeh, D. W. (2006). Male-killing Wolbachia in a livebearing arthropod: brood abortion as a constraint on the spread of a selfish microbe. J Invertebr Pathol 92, 33-38.

Zeh, D. W., Zeh, J. A. \& Bonilla, M. M. (2005). Wolbachia, sex ratio bias and apparent male killing in the harlequin beetle riding pseudoscorpion. Heredity 95, 41-49.

Zhang, K., Martiny, A. C., Reppas, N. B., Barry, K. W., Malek, J., Chisholm, S. W. \& Church, G. M. (2006). Sequencing genomes from single cells by polymerase cloning. Nat Biotechnol 24, 680-686.

Zhou, W., Rousset, F. \& O'Neil, S. (1998). Phylogeny and PCR-based classification of Wolbachia strains using wsp gene sequences. Proc Biol Sci 265, 509-515.

Edited by: A. Holmes 